Acquisition et interaction en langue étrangère

$13 \mid 2000$

$\mathrm{La}$ Lecture en langue étrangère

\title{
Lecture de textes en le et compétence textuelle
}

\section{Marc Souchon}

\section{OpenEdition}

\section{Journals}

\section{Édition électronique}

URL : https://journals.openedition.org/aile/1462

DOI : 10.4000/aile.1462

ISSN : 1778-7432

Éditeur

Association Encrages

Édition imprimée

Date de publication : 1 septembre 2000

Pagination : 15-40

ISSN : 1243-969X

\section{Référence électronique}

Marc Souchon, «Lecture de textes en le et compétence textuelle», Acquisition et interaction en lanque étrangère [En ligne], 13 | 2000, mis en ligne le 13 décembre 2005, consulté le 14 avril 2022. URL : http:// journals.openedition.org/aile/1462 ; DOI : https://doi.org/10.4000/aile.1462

Ce document a été généré automatiquement le 14 avril 2022.

(c) Tous droits réservés 


\title{
Lecture de textes en le et compétence textuelle
}

\author{
Marc Souchon
}

1 Affirmer qu'il peut y avoir lecture de textes dans les premières phases d'apprentissage d'une LE, soulève de très nombreuses questions au point d'ailleurs que le terme de lecture n'est peut-être pas le plus satisfaisant. Nous n'entrerons pas ici dans cette discussion. Nous ne reviendrons pas non plus sur l'hypothèse de J.C. Alderson selon laquelle « une sorte de seuil ou de plafond de compétence langagière doit être atteint avant que les habiletés existant en L1 puissent être transférées à la L2 » (cf. Alderson \& Urquhart, 1984). Nous considérerons, de façon quelque peu hasardeuse, que les lecteurs qui nous ont servi d'informateurs l'ont franchi.

2 Nous centrerons nos analyses sur ce que ces lectures révèlent de la compétence textuelle acquise par le sujet. Nous estimons en effet que pendant les débuts de l'apprentissage d'une LE, l'ensemble des expériences de la textualité sur lequel l'apprenant s'appuie joue un rôle déterminant au cours des premières lectures en LE.

1. Définitions

3 Il n'est pas question de développer ici une théorie du texte. Nous nous appuierons essentiellement sur l'appareil notionnel proposé par J.-P. Bronckart (1996) dans le cadre d'un interactionnisme socio-discursif. Cet auteur distingue nettement trois niveaux d'approche :

4 (1) Les activités langagières à l'œuvre dans les collectivités humaines [...] qui se déploient dans le cadre de formations socio-langagières [...] auxquelles participent des agents singuliers, en tant que sièges d'actions langagières déterminées. (2) Les textes, en tant que formes communicatives globales et 'finies' constituant les produits concrets des actions langagières [...] qui se distribuent en genres adaptés aux enjeux des formations socio-langagières; ces genres, dont le nombre et les frontières sont par essence indéterminés, restant à disposition au titre de modèles dans ce construit sociohistorique qu'est l'intertexte. (3) Les types de discours, comme formes linguistiques attestables dans les textes et traduisant la création de mondes discursifs spécifiques (p. 150-151, les caractères gras et les italiques sont dans le texte). 
Un texte singulier est donc une 'entité empirique' dans laquelle se matérialise l'action langagière imputable à un agent (p. 39).

6 Nous considérerons que la compétence textuelle se met en place à partir des expériences du sujet liées à sa fréquentation des textes. Elle présente des dimensions à la fois individuelles, sociales et culturelles et peut être décomposée en un ensemble de savoirs, de savoir-faire et de représentations (notre définition du terme compétence rejoint ainsi celle donnée par M. Dabène 1991 : 26). L'école joue sans doute un grand rôle dans la manière dont se développe cette compétence textuelle mais il serait certainement faux de prétendre qu'elle dépend uniquement de la scolarisation du sujet. En situation LE, si la compétence textuelle que le sujet a acquise est susceptible, dans certains cas, de lui fournir une aide, on peut également penser qu'elle va progressivement se transformer à travers la fréquentation de textes produits dans une autre langue-culture. La compétence textuelle n'est pas figée; elle présente au contraire un caractère évolutif en fonction du vécu de chacun, la lecture en LE faisant pour nous partie des expériences privilégiées.

7 Concernant l'activité de lecture, on peut la décrire comme l'ensemble des processus de déconstruction/reconstruction à travers lesquels le texte va progressivement s'intégrer au discours propre du lecteur. La dynamique interactionnelle entre le pôle émetteur et le pôle récepteur qui se développe à partir de la mise en place de la situation de lecture fait que l'objet sémiotique constitué par le texte se transforme chez le lecteur en discours intérieur, et en se transformant en discours intérieur, va modifier ce discours intérieur lui-même.

8 Le texte agit en profondeur sur le rapport du sujet au langage : dans le texte, le langage est à la fois une trace matérielle inscrite sur un support (un objet parmi d'autres objets, une image parmi d'autres images), et un discours qui s'adresse à un sujet, qui lui parle pour entrer en résonance avec son propre discours. Avec le texte, le sujet est amené à accepter cette double nature du langage et à établir des relations entre ces deux ordres de manifestation dans des situations de communication particulières. Si cette expérience n'est pas résolue de façon satisfaisante par le sujet, il y a fort à parier que le texte ne deviendra pas pour lui un lieu où se joue la question du sens. Cette spécificité du texte fait qu'il peut y avoir des formes de déliaison entre le 'langage objet' et le langage intérieur du sujet.

9 Dans les limites de cet article, nous nous intéresserons uniquement à trois aspects de la compétence textuelle :

10 - la question du genre ${ }^{1}$ qui nous paraît être primordiale du point de vue de la compréhension. Il semblerait en effet qu'une des premières préoccupations du lecteur confronté à un texte soit de le rattacher à un genre ou à une catégorie textuelle qu'il connaît déjà ;

11 - l'introduction dans le texte d'une parole autre que celle de l'énonciateur 1 (ou narrateur). Il s'agit là d'un phénomène énonciatif/discursif puisqu'il suppose l'introduction d'autres voix dans le texte et par conséquent, d'un discours autre. Il est aussi de nature interactive étant donné que toute introduction d'un discours autre entraîne une évaluation réciproque des discours en présence. Il est enfin textuel, dans la mesure où le rapport de ce que Peytard (1968) appelle le 'verbal' et le 'non verbal' se joue d'une façon particulière dans chaque texte. 
12 Nous le ferons en nous appuyant à la fois sur des exemples de textes littéraires ou non littéraires, ce qui nous amènera à aborder un troisième aspect de la compétence textuelle, celui de l'existence du fait littéraire. Nous considérons en effet que le littéraire doit être pris en compte dans une réflexion sur la compétence textuelle. À des degrés certes très divers, il entre dans l'expérience de la textualité acquise par un sujet.

2. La question du genre (Expérimentation 1)2.1. Recueil des données

Constitution d'une micro-anthologie de 14 textes (ou extraits de textes) organisée autour de l'opposition entre textes fictionnels-littéraires ou 'TEXTES' et textes non fictionnels - non littéraires 'DOCUMENTS' (J. Peytard \& S. Moirand 1992: 61)². Concernant l'opposition FICTIONNEL/ vs/NON FICTIONNEL, elle est liée à la capacité du langage de pouvoir engendrer d'autres univers en dehors de ceux que nous connaissons à travers notre expérience du quotidien. Quant à l'opposition TEXTE/vs/ DOCUMENT, elle recoupe l'opposition littéraire/vs/non littéraire; elle est donc de nature plutôt sociale et culturelle. Si la plupart des textes qualifiés de littéraires relèvent de la fiction, on ne peut cependant pas considérer que les catégories du fictionnel et du littéraire soient équivalentes.

Nous avons les deux sous-ensembles textuels (ET) suivants :

- ETA = ‘TEXTES' : 10 extraits de textes littéraires

16 - ETB = 'DOCUMENTS' : 4 articles journalistiques du type faits divers/faits de société . $^{\text {. }}$

17 En dehors d'un exemple de récit à la troisième personne et au passé simple que nous avons nous-même sélectionné, les 10 textes de ETA font partie de l'opuscule de E. Wagner (1965): De la langue parlée à la langue littéraire. On a au total 5 récits, 3 textes descriptifs, 1 texte argumentatif et 1 poème ${ }^{4}$.

$\begin{array}{ll}\text { Entre ETA et ETB, il existe les oppositions suivantes : } \\ \text { ETA } & \text { vs ETB } \\ \text { fictionnel } & \text { vs non fictionnel } \\ \text { littéraire } & \text { vs non littéraire } \\ \text { extraits de textes plus longs } & \text { vs textes complets } \\ \text { coupures opérées dans les textes } & \text { vs textes non modifiés }\end{array}$

Cette micro-anthologie a été présentée aux étudiants en trois fois. ETA a été divisé en deux séries de cinq textes chacune. Les 'documents' ETB ont été lus en une seule séance; chaque groupe d'informateurs n'a eu à lire que deux documents (sur les quatre) afin de réduire la quantité de lecture.

Observables : les soulignements. Les étudiants devaient souligner dans les textes «ce qui faisait obstacle à la progression de leur lecture ", qu'il s'agisse de lexèmes isolés ou d'unités plus larges pouvant aller jusqu'à des macro-unités du texte. On a donc utilisé le sentiment qu'ont les lecteurs de comprendre ou de ne pas comprendre. Les soulignements ne rendent pas compte de l'activité de lecture dans son déroulement effectif: souvent, les étudiants ne soulignent un lexème ou un passage du texte qu'après être arrivés à la fin d'une unité plus large, phrase, paragraphe, voire même la fin du texte.

20 Par rapport à ces observables que sont les soulignements :

$211^{\circ}$ nous estimons qu'ils n'expriment pas directement le fait de comprendre ou de ne pas comprendre, ce qui serait une interprétation par trop schématique, sans grand intérêt par rapport à une analyse des processus de compréhension. Pour nous, les 
soulignements sont avant tout l'indication d'une attention particulière portée à telle unité du texte (faut-il y voir la manifestation d'un surcroît de traitement de la part du sujet ?).

$2^{\circ}$ Ce qui nous intéresse dans l'analyse des soulignements, c'est de localiser les zones du texte où ils s'accumulent. On obtient une sorte de carte du texte correspondant aux lieux où se concentreraient les difficultés du point de vue de la reconstruction du sens.

2.2. Résultats2.2.1. L'incidence du niveau en LE et de la langue dominante des informateurs

Niveau en LE

Les 220 étudiants ont été répartis en trois groupes en fonction de la durée de leur apprentissage du français : groupe 1 (34\%), de 3 à 4 semestres; groupe $2(50,4 \%)$, de 5 à 8 semestres; groupe $3(15,5 \%)$, au delà de 8 semestres 5 .

D'une façon générale, l'incidence de la maîtrise de la LE n'est pas aussi évidente qu'on aurait pu le supposer. Il n'est pas possible non plus d'établir de relation claire entre le comportement des étudiants de niveau avancé et la difficulté des textes appréciée à la fois à travers le pourcentage de soulignements et à partir de l'évaluation des étudiants eux-mêmes. Il semblerait même que lorsque cette difficulté augmente, le comportement des trois groupes ne se différencie plus.

\section{Langues représentées}

Les informateurs se répartissent de la manière suivante: langues latines (espagnol, italien et portugais) $32 \%$; anglais y compris pays africains anglophones $20 \%$; allemand $18,5 \%$; arabe $8,5 \%$; japonais $7 \%$.

Dans la moitié des cas seulement le comportement des lecteurs de langue latine se différencie nettement de celui des autres groupes linguistiques. On observe par ailleurs que les lecteurs arabophones et japonophones se retrouvent toujours du même côté du graphe et dans des zones très voisines. Les comportements de ces deux sous-groupes de lecteurs tendent donc à se rapprocher mais ils ne s'opposent jamais totalement à ceux des autres groupes linguistiques.

Par rapport à ces 14 textes, il est impossible de dégager un comportement spécifique à un groupe linguistique représenté. Il n'est pas non plus possible d'établir une relation entre genre de textes et groupe linguistique.

2.2.2. L'incidence du genre observée à partir du traitement du matériau verbal dans les textes

30 Nous nous sommes basé sur les variations des pourcentages de mots grammaticaux, de substantifs, d'adjectifs et de verbes dans les textes et dans les soulignements.

On constate que dans les textes, le pourcentage de mots grammaticaux est relativement stable. Pour les 14 textes retenus, il oscille entre 41 et $53 \%$. Par rapport aux autres catégories de lexèmes, il s'agit effectivement de la plus stable. Concernant ces autres catégories, on observe pour les textes de la sélection une sorte de règle générale. Selon la catégorie à laquelle le texte se rattache, les pourcentages de substantifs, d'adjectifs, de verbes et d'adverbes varient d'une façon somme toute assez prévisible :

Pour les récits on $a$ : Verbes $>$ Substantifs $>$ Adjectifs

Pour les textes descriptifs : $\mathrm{A}>\mathrm{S}>\mathrm{V}$

Pour les textes journalistiques $: \mathrm{S}>\mathrm{V}>\mathrm{A}$ 

ailleurs, bien des points mériteraient d'être discutés. Mais, ce qui nous intéresse audelà des pourcentages eux-mêmes, c'est de constater :

Si l'on regarde maintenant les soulignements, il apparait :

- qu'en ce qui concerne les mots grammaticaux, leur pourcentage est soumis à des variations plus fortes. Il reste en général faible mais, dans certains cas, il devient assez significatif : on passe ainsi de 9 à $26 \%$.

- Pour les autres catégories de lexèmes, l'écart entre les pourcentages se creuse mais pour les 5 récits et les 3 textes descriptifs, les valeurs atteintes respectent le même classement.

Récits : V > S > A

Textes descriptifs : $\mathrm{A}>\mathrm{S}>\mathrm{V}$

On peut par conséquent dire que pour ces deux catégories textuelles, les soulignements amplifient, parfois de manière assez sensible, les phénomènes observables au niveau de la répartition initiale des lexèmes sans toutefois la remettre en cause.

Nous avons constaté la même homogénéité pour les quatre textes journalistiques du type faits divers/faits de société mais avec cependant une variante : du point de vue de la répartition des différentes catégories de lexèmes, ils apparaissent groupés (ce qui tendrait à prouver qu'ils constituent une catégorie textuelle à part). Lorsqu'on regarde ensuite ce qui se passe du côté des soulignements, il se produit un phénomène identique à savoir que s'opère une modification de la répartition initiale entre les différentes catégories de lexèmes :

Répartition initiale des lexèmes dans les 4 textes journalistiques :

$\mathrm{S}>\mathrm{V}>\mathrm{A}$

Répartition des lexèmes dans les soulignements effectués :

$\mathrm{V}>\mathrm{S}>\mathrm{A}$

Les résultats d'une étude aussi limitée ne sont certainement pas généralisables. Par $1^{\circ}$ que des paramètres tels que le niveau en LE ou la langue d'usage sont peu discriminants ;

$2^{\circ}$ qu'en revanche, on observe des sortes de régularités lorsqu'on se situe du point de vue du genre (ou de la catégorie des textes), même si les exemplaires singuliers qui les représentent dans cette micro-anthologie sont sensiblement différents les uns des autres (ce qui est le cas des 5 récits, des 3 textes descriptifs et des 4 textes journalistiques).

49 Ces résultats tendraient à montrer que la catégorie du texte influe en profondeur sur la nature des traitements opérés. Ils pourraient servir d'argument pour étayer l'hypothèse selon laquelle les différences entre les genres seraient à ce point intériorisées qu'elles opéreraient une sorte de guidage de la lecture. Bien entendu, ce degré d'intériorisation des différences entre les catégories de textes reste un point en discussion.

3. L'introduction dans le texte d'une parole autre

50 Pour aborder cette question, nous utiliserons deux sortes de données : d'une part, celles recueillies dans le cadre de l'Expérimentation 1 et d'autre part, des corpus constitués à partir d'activités de type rappel ou résumé de texte (Expérimentations 2 et 3).

Acquisition et interaction en langue étrangère, 13 | 2000 
51 Dans un premier temps, nous reviendrons sur l'analyse de la localisation des soulignements en prenant l'exemple de deux récits de la micro-anthologie (sousensemble ETA) où le discours rapporté est présent. Puis nous étudierons l'incidence du discours rapporté sur les épreuves de type rappel ou résumé de texte: dans l'expérimentation 2, il s'agira d'un fait divers journalistique et dans l'expérimentation 3 d'un récit littéraire.

3.1. Localisation des soulignements dans deux récits de ETA où se manifeste le discours rapporté

52 Sur les quatre textes concernés, nous commenterons uniquement ce qui se passe dans le cas de deux récits (TF8 et TF16 présentés en annexe) qui constituent à nos yeux deux exemples particulièrement significatifs.

Dans TF8 (Maman et son dîner) ${ }^{6}$ le discours direct occupe une grande place (voir sur le plan scripto-visuel, l'utilisation de guillemets et de tirets pour signaler les tours de parole). Cependant sur le plan sémantique, le dit est d'emblée dévalorisé. La mère, qui est ici le personnage principal, distribue la parole aux autres membres de la famille selon un ordre très ritualisé mais en sachant que ces prises de parole successives ne régleront pas le problème (voir $\mathrm{P} 10$ ). Une hiérarchie très nette s'établit entre le personnage principal, celui qui fait, et les autres 'qui ne font que parler'.

54 Pour ce qui est des soulignements, on pourrait s'attendre à ce qu'ils portent sur le discours direct et notamment sur le vocabulaire de la nourriture ${ }^{7}$. D'une manière générale, ce n'est pas ce que l'on observe : seul « noix » apparaît au 7e rang. Pour ce qui est du discours direct, en dehors du cas de " tué »(4e rang), les soulignements s'accumulent plutôt sur les éléments entourant les prises de parole et principalement sur la résolution (P18). On remarquera que ces éléments (" debout », « en sautant », « elle a déjà empoigné le panier fermé, en rotin noir ») correspondent à la fois à la reprise du récit et à un commentaire (qui est aussi une évaluation) de ce qui vient d'être dit : dire " debout " (la mère) et dire " en sautant en l'air » (la fille) sont deux manières de dire qui sont ici opposées; quant à la fin (P18), elle constitue en elle-même une évaluation du point de vue de la mère, de la scène qui vient de se passer.

55 À l'exception de P1 (pour des raisons que nous ne développerons pas ici), ce n'est pas sur le dit (le verbal) que l'attention des lecteurs semble s'être portée au cours de la reconstruction du sens de TF8 - ces éléments verbaux étant par ailleurs présentés comme sans importance dans le texte.

56 En ce qui concerne TF16 (Projets d'avenir) ${ }^{8}$, le discours indirect domine tout au long du texte en dehors de P7 où le discours direct apparaît. Le dire a ici une importance capitale et c'est naturellement la réponse du narrateur, personnage principal, qui est attendue par le lecteur; attente du reste minutieusement ménagée par le travail de l'écriture. C'est en P10 en effet qu'il se définit lui-même en tant que personnage et cette prise de position est essentielle pour le lecteur parce qu'elle lui permet de comprendre à quel type de héros romanesque il a affaire. Il s'agit par conséquent d'une déclaration dont la fonction d'anticipation est tout à fait considérable puisqu'elle s'applique à l'ensemble des actions qui vont être racontées par la suite. Elle peut être discutée au plan psychologique si l'on estime que les personnages des romans sont aussi 'des personnes de chair et de sang' mais elle annonce en même temps une sorte de programme narratif. Il est évident que l'évaluation par le patron des propos tenus par le personnage principal est tout aussi importante pour la compréhension de P10; et qu'avec P11 on entre dans ce jeu d'évaluation réciproque des discours dont nous avons 
parlé ci-dessus : chaque nouvelle manifestation du discours rapporté se développe tout en portant évaluation des paroles précédemment attribuées à l'autre personnage sans que jamais on ne sorte de la perspective du narrateur.

Il est intéressant de constater que les soulignements s'accumulent précisément sur ces deux zones du texte; d'abord sur P10 et ensuite sur P11, comme si les soulignements établissaient une sorte de hiérarchie entre les deux. Pour P10, il apparaît que des mots grammaticaux comme 'la mienne viennent au deuxième rang, ce qui est assez exceptionnel. Or on ne peut certainement pas dire que le mot 'vie' soit un mot inconnu des étudiants ni qu'il puisse y avoir hésitation sur le référent repris par 'la mienne'. 'Vie' est en effet répété trois fois entre P7 et P10 sans compter une occurrence de 'vivre' en P6. Pour P11, les soulignements se portent sur les deux adjectifs où l'on pourrait dire que se concentre la fonction évaluative des propos du patron, à savoir 'mécontent' et 'désastreux'.

Dans ces deux textes où il y a présence du verbal dans le récit, on observe un déplacement des accumulations de soulignements qui, selon les cas, se portent soit sur les éléments du verbal lui-même soit sur les éléments qui l'entourent et servent à le mettre en scène. Cela signifie pour nous qu'il y a une dimension textuelle des obstacles signalés par les apprenants au cours de leur lecture. Encore une fois, on peut légitimement s'interroger sur le fait de savoir si cette observation est généralisable ou si elle ne s'expliquerait pas plutôt par certaines caractéristiques des récits de la microanthologie (notamment le fait qu'il s'agit de textes relativement courts). Pour répondre à cette question d'autres enquêtes seraient nécessaires. Il n'en demeure pas moins qu'un phénomène discursif tel que l'irruption du verbal dans un récit ne peut être ignoré lorsqu'on essaye de réfléchir sur la compréhension des textes narratifs. Nous avons là un exemple parmi d'autres de ces phénomènes liés à la mise en texte d'un récit, inhérent par conséquent à l'écriture narrative elle-même, qu'un lecteur expert est habitué à rencontrer dans sa LM.

Il convient cependant de ne pas perdre de vue que ces phénomènes de polyphonie se manifestent dans chaque texte de façon particulière. On est là par conséquent du côté de la singularité de la relation émetteur - texte - lecteur. Les différentes voix, les différents discours se font écho l'un à l'autre dans un jeu d'évaluations réciproques construit par le texte auquel le lecteur est invité à prendre part. Sa propre voix va se construire en résonance avec les autres.

Pour terminer sur ce point, il convient d'insister sur la double orientation prise par nos analyses. D'un côté, au niveau global, des observations allant dans le sens d'un traitement homogène de textes appartenant à une même catégorie ; à l'autre extrémité, des observations faisant apparaitre une spécificité irréductible de chaque texte, une sorte d'empreinte du texte. Cela va parfaitement dans le sens des considérations de J.-P. Bronckart (1994: 378) à propos de la double nature du genre :

61 L'homme ne s'exprime qu'en produisant du 'texte' relevant d'un genre particulier [...] Chaque exemplaire d'un genre [...] peut être considéré comme une unité autosuffisante, en ce qu'elle constitue la concrétisation verbale d'une unité comportementale, ou encore la concrétisation d'une action langagière définie.

Si l'on se situe dans le cadre de la psychologie cognitive, les phénomènes de ce type feraient partie des informations de haut niveau apportées par le texte et entreraient dans les connaissances préalables dont dispose le sujet (Gaonac'h 1987 : 164 -167). Notre hypothèse va dans le même sens: les déplacements au niveau des accumulations de 
soulignements manifesteraient l'existence chez les sujets de l'enquête d'acquis antérieurs liés à la fréquentation des écrits. Pour être plus précis, l'expérience des informateurs concernant la catégorie récit, présente à la fois une dimension discursive dans la mesure où la relation entre le verbal et son entour est pour eux une source d'interrogations et textuelle dans la mesure où leur questionnement est orienté différemment selon les textes. A propos de cette dernière, l'expérimentation 1 montrerait en outre que le texte est à la fois exploré en tant qu'appartenant à un genre et à la fois en tant qu'exemplaire unique de ce genre. Pour nous la mise en œuvre de ces différents niveaux d'approche constituerait une manifestation de la compétence textuelle acquise, à cette étape de son développement.

3.2. Les incidences de la présence du discours rapporté dans les épreuves de type rappel ou résumé de texte3.2.1. Expérimentation 2

\section{Recueil des données}

Nous nous sommes appuyé sur une enquête effectuée par Orapin Kampanthong dans le cadre d'une thèse de Doctorat soutenue en 1993 à Grenoble III ${ }^{9}$.

Le texte retenu est paru dans un hebdomadaire politique thaïlandais du type Nouvel Observateur (p. 295).

Ce texte a été donné à lire à 3 groupes désignés par A, B, C, les groupes $\mathrm{A}$ et $\mathrm{B}$ étant chacun constitué de 25 étudiants thaïlandais et le groupe $C$ de 25 étudiants français.

Lecture en LM : le groupe A lit le texte en thaï ; le groupe $C$ lit la traduction en français de ce même texte. Lecture en LE : le groupe B (étudiants thaïlandais ayant le français comme matière principale), lit la traduction en français du texte ; le niveau en LE de ce groupe pourrait être qualifié d"intermédiaire‘.

\section{Consignes}

- Les étudiants qui lisent le texte en LM (groupes A et C) ont 10 minutes pour le faire. Ceux qui le lisent en LE (groupe B) disposent de 20 minutes.

- Passé ce temps, le texte leur est retiré. Ils doivent alors écrire 'tout ce qu'ils ont compris dans le texte' (p.296). Aucune limitation de temps n'est imposée pour cette deuxième partie de l'expérience. Les productions écrites obtenues en thaï pour les groupes $\mathrm{A}$ et $\mathrm{B}$ ont été traduites en français par $\mathrm{O}$. Kampanthong ce qui pose à nouveau le problème de la traduction.

1 Analyse du texte

Le texte retenu est de type fait divers. Les faits rapportés sont situés en Autriche, hors $\mathrm{du}$ 'territoire culturel' propre à des lecteurs thaïs ou français.

73 L'analyse rapide que nous allons effectuer est essentiellement basée sur la traduction de 0 . Kampanthong ${ }^{10}$. Le titre du texte est une expression thaïe utilisée pour désigner deux personnes qui s'affrontent sans cesse et qui ne se mettront jamais d'accord. On pourrait le traduire par : 'des adversaires irréductibles'. Ce titre, vu ce qui suit, annonce donc la dimension humoristique du texte.

74 La relation scripteur-destinataire est fortement marquée. Elle encadre pour ainsi dire le texte. Au début, les guillemets (qui apparaissent également dans le texte thaï), signalent 'un proverbe' renvoyant à la tradition bouddhiste. Le scripteur détourne ce 'proverbe' de sa forme originale qui serait 'Les chiens nous mordent, il ne faut pas les mordre'. À la fin, le dernier paragraphe opère un retour à cet univers culturel, le 
lecteur thaï étant directement pris à parti. L'humour se manifeste également au $\S 4$ : la traduction exacte serait non pas 'facteurs' mais 'nos jeunes livreurs de courrier'.

La polyphonie énonciative. À partir de la phrase 4 (P4) et jusqu'à P11, l'énonciateur 'se met en retrait' (en dehors des modalités des lignes 8 et 17), cédant la place à d'autres voix : 'les statistiques' $(\$ 1,1.5)$; 'l'agence de presse Reuter' $(1.10)$. On a affaire à une succession très réglée d'énonciateurs.

À propos de la relation scripteur-lecteur, on observera :

$-1^{\circ}$ l'écart existant entre le nombre de rappels de P1 et de P11. En effet pour P1, s'agissant d'une référence à la sagesse bouddhique, il paraît assez normal de trouver dans les rappels thaïs un nombre important de reprises (ce qui est confirmé : A = 15 rappels $/ 25>C=8 / 25)$. En revanche, pour P11 $(\$ 4)$ qui opère pourtant un retour à la situation de la Thaillande, on $\mathrm{a}: \mathrm{A}=3 / 25<\mathrm{C}=12 / 25$. Le fait de se situer à l'intérieur de la culture d'origine des lecteurs ne permet pas d'expliquer le faible nombre de reprises de P11 dans le cas du groupe A.

Tableau 1

\begin{tabular}{|l|l|l|l|}
\hline & \multicolumn{2}{|l|}{ Lecteurs thais } & Lecteurs français \\
\hline & $\begin{array}{l}\text { Groupe A } \\
\text { LM }\end{array}$ & $\begin{array}{l}\text { Groupe B } \\
\text { LE }\end{array}$ & $\begin{array}{l}\text { Groupe C } \\
\text { LM }\end{array}$ \\
\hline $\begin{array}{l}\text { § 1 Rappels partiels } \\
\text { + rappels intégraux }\end{array}$ & 25 & 25 & 25 \\
\hline $\begin{array}{l}\text { § 2 Rappels partiels + } \\
\text { rappels inté graux }\end{array}$ & 24 & 19 & 22 \\
\hline $\begin{array}{l}\text { § 3 Rappels partiels + } \\
\text { rappels intégraux }\end{array}$ & 16 & 17 & 23 \\
\hline $\begin{array}{l}\text { § 4 Rappels partiels } \\
\text { + rappels intégraux }\end{array}$ & 3 & 3 & 13 \\
\hline
\end{tabular}

$2^{\circ}$ La nature des rappels de P1 effectués par le groupe A. Deux tendances principales se dégagent: les rappels où il $\mathrm{y}$ a une forme de commentaire de la 'citation' pour bien marquer son sens véritable : « cette expression ne veut pas dire qu'il faut mordre les chiens mais... "; d'autre part ceux, relativement moins nombreux, où il s'agit de reprises qui, d'une façon ou d'une autre, rapportent en fait 'le proverbe' aux facteurs : « les chiens mordent les facteurs; ces derniers doivent à leur tour les mordre/les corriger $»$.

80 Pour les locuteurs thaïs que nous avons consultés, le début de ce texte poserait un problème lié à la citation du 'proverbe' bouddhiste et par conséquent à un phénomène de polyphonie énonciative : dans quelle mesure a-t-on le droit de se référer à la sagesse traditionnelle pour parler de quelque chose de 'trivial'?

81 À propos des autres voix du texte (les statistiques, l'Agence Reuter), elles sont reprises de façon très stable dans tous les rappels, qu'il s'agisse des groupes A, C ou B. Les différences de langue-culture semblent ici sans effet. D'autre part, il apparaît que si la longueur des rappels reste toujours inférieure à celle du texte initial, cette réduction ne se produit pas au détriment de la référence aux différents hétéro-énonciateurs. 

polypohonie en tant que tel qui a une incidence sur les rappels mais plutôt la charge culturelle (et ici religieuse) du discours cité (voir $\$ 1$ du texte).

\subsubsection{Expérimentation 3}

texte dans le verbal à partir de $\mathrm{P} 6$. Selon le schéma narratif canonique à cinq phases, l'action correspond à l'irruption dans le récit du discours rapporté. On a donc là une 'entaille' majeure (Peytard et al. $1982: 144$-150) liée au passage du récit des actions au discours rapporté. Il convient cependant de noter que le verbal apparaît dès P2, 1.2 « se convenció de que... ", qu'il se manifeste à nouveau en P4 1.5 " Se dijo... » Nous appellerons MU1 la première macro-unité de ce texte dominée par le récit des actions (de P1 à P5, 1. 1-10) et MU2 la deuxième (de P6 à P9, 1. 10-23). Si l'on s'en tient au seul rapport MU1/MU2, la deuxième macro-unité textuelle est plus développée que la première (de $\mathrm{P} 1$ à $\mathrm{P} 5,115$ lexèmes, soit $39 \%$; de $\mathrm{P} 6$ à $\mathrm{P} 9,177$ lexèmes, soit $61 \%$ ) et si l'on prend en compte les manifestations du verbal présentes dans MU1, c'est bien le verbal qui l'emporte incontestablement dans l'ensemble de ce texte.

(2) La fin du récit. La dernière phrase prononcée par le réceptionniste ne met pas un terme à la quête de la chambre entreprise par Sebastián, le personnage principal. Celleci est simplement différée sans que l'on puisse rien dire de son résultat. Par conséquent, ce texte ne fait pas avancer l'action. Le dynamisme discursif est donc plutôt du côté du discours rapporté (ou du verbal).

Dans un texte narratif comme celui-ci le discours rapporté est premier et sa fonction semble être à la fois de suspendre l'action et de susciter un monde de fiction qui finit par se superposer à la fiction première née de la narration. On a affaire à une sorte de construction en abîme où le récit fictionnel crée à son tour une autre fiction née de la parole attribuée aux personnages, parole proférée dans le cas du dialogue entre Sebastián et le réceptionniste, ou simplement pensée (du type discours intérieur, dans le cas des manifestations du verbal disséminées dans MU1). La tension majeure de ce texte n'est donc pas liée au dynamisme narratif mais à ce rapport entre verbal et non verbal.

Résultats $^{12}$

En LE, on obtient un faible pourcentage de fins conformes (31\%) exprimant clairement le fait que l'issue de la quête de la chambre est différée. Lorsqu'on passe de la situation LM à la situation LE, on observe que l'écart le plus important concerne le pourcentage d'issues négatives de la quête (Sebastián ne trouve pas de chambre) : LM 5,71 \%, LE $23,94 \%$ alors que le pourcentage d'issues positives reste plutôt stable : LM 11,42 \%, LE 
$12,66 \%$. Que certains élèves (17,13 \% en LM contre $36,6 \%$ en LE), aient éprouvé le besoin de terminer leur résumé de façon positive ou négative, nous paraît correspondre à une certaine représentation du schéma narratif conçu comme une simple tension entre une complication initiale et une résolution. On pourrait dire alors que la situation LE accentue les effets de cette représentation. Mais cela n'explique pas qu'en LE, l'issue négative soit plus représentée qu'en LM, d'autant que la fin du texte laisse plutôt augurer une issue positive (voir la promesse du réceptionniste).

Tableau 2

\begin{tabular}{|l|l|l|}
\hline & $\begin{array}{l}\text { LM - 105 } \\
\text { résumés }\end{array}$ & $\begin{array}{l}\text { LE - 71 résumés } \\
\text { (NB 5 résumés ne sont } \\
\text { pas terminés) }\end{array}$ \\
\hline $\begin{array}{l}\text { État final non exprimé ou } \\
\text { problématique } \\
\text { (Etat final ?) }\end{array}$ & $\begin{array}{l}4 \\
(3,8 \%)\end{array}$ & $\begin{array}{l}23 \\
(32,40 \%)\end{array}$ \\
\hline $\begin{array}{l}\text { SE trouve une chambre } \\
\text { (Issue positive) }\end{array}$ & $\begin{array}{l}(2,42 \%) \\
(11,42 \%)\end{array}$ & $\begin{array}{l}9 \\
(12,66 \%)\end{array}$ \\
\hline $\begin{array}{l}\text { SE ne trouve pas de chambre } \\
\text { (Issue négative) }\end{array}$ & 6 & 17 \\
$(5,71 \%)$ & $(23,94 \%)$ \\
\hline $\begin{array}{l}\text { Etat final non conforme } \\
\text { Sous total }\end{array}$ & $\begin{array}{l}22 \\
(20,93 \%)\end{array}$ & $\begin{array}{l}49 \\
(69 \%)\end{array}$ \\
\hline $\begin{array}{l}\text { L'issue de la quête est différée } \\
\text { (État final conforme) }\end{array}$ & 83 & $\begin{array}{l}22 \\
(31 \%)\end{array}$ \\
\hline
\end{tabular}
de l'état final. Autrement dit, il semble bien que dans un texte comme celui-ci, on ne puisse pas dissocier la reconstitution de la séquence d'actions de la reprise de MU2. Il n'y pas de divergence entre ce qui est observable en LM et en LE, la situation LE ne faisant qu'accentuer certaines tendances déjà repérables en LM. Il n'en reste pas moins vrai que pour nous, ce sont bien les difficultés rencontrées au niveau de la reprise de MU2 qui expliqueraient l'augmentation du pourcentage d'issues non conformes en situation LE ainsi que la différence entre le pourcentage d'issues négatives en LM et en LE.

4. Le littéraire et la compétence textuelle

lexpérimentation 1 , nous avons constitué une micro-anthologie à partir de la distinction opérée par Peytard et Moirand (1992 : 61) entre TEXTE et DOCUMENT, deux catégories de productions textuelles qui se situeraient pour ces auteurs entre deux pôles extrêmes: d'un côté, le THEOREME (fondamentalement monosémique); de l'autre, le POEME (fondamentalement polysémique). 

considère ces deux catégories comme deux ensembles polairement opposés :

\section{TEXTE vs DOCUMENT}

Celle de M. Dabène (1991) qui voit les choses plutôt sous la forme d'« un continuum scriptural ». A ses yeux, même les écrits « ordinaires » s'inscrivent pour le scripteur dans une situation de communication vécue par lui comme de fait « extraordinaire ».

C'est en fait tout le problème de la spécificité du littéraire qui est posé ici. Dans nos propres expérimentations, nous nous efforçons de faire alterner les textes littéraires avec les autres. Pour cela nous sélectionnons des textes reconnus comme littéraires, produits par des écrivains de profession. Nous nous situons par conséquent plutôt du côté de l'opposition Littéraire vs Non littéraire plutôt que du continuum.

Afin d'apporter quelques éléments de discussion sur cette question, nous reviendrons sur les Expérimentations 1 et 3.

\subsection{Retour sur l'Expérimentation 1}

100 Nous avons remarqué dans notre enquête statistique sur les soulignements que le seul texte poétique de la micro-anthologie (il s'agit du poème de Prévert : Le cancre) occupe une position tout à fait à part par rapport aux autres ${ }^{13}$. Globalement, si l'on considère le sous-ensemble ETA (voir ci-dessus en 2.1), il vient au deuxième rang en ce qui concerne le pourcentage de soulignements ${ }^{14}$ (si l'on s'en tient strictement à la sélection proposée dans l'ouvrage de E. Wagner 1965, il figure en tête). De plus, on observe que ces soulignements ont été effectués par le pourcentage le plus élevé de lecteurs de niveau le plus avancé. Par ailleurs, on a affaire à un exemple dans lequel les mots grammaticaux viennent en tête : 'soudain' 4e rang, 'malgré' 8e rang. Par rapport à tous les textes de la micro-anthologie, ce sont l'adverbe et la préposition qui occupent le rang le plus élevé. Il s'avère par ailleurs qu'un mot appartenant au vocabulaire usuel de l'apprenant comme 'craie' vient au 3e rang. Enfin, c'est dans ce texte que les soulignements sur le titre sont les plus nombreux alors que pour les autres, c'est une zone qui ne semble pas avoir retenu l'attention des informateurs.

Nous estimons que les réactions, très sensibles au plan quantitatif, observées à propos de ce texte s'expliquent par le fait poétique lui-même (sans doute ressenti d'autant plus fortement par les lecteurs qu'il s'agit en fait du seul poème figurant dans le sousensemble ETA). Le lecteur est mis en présence d'une écriture fondamentalement polysémique, d'une pluralité de sens à la fois ouverte et offerte par un texte disposé en outre verticalement. Tout cela contribue sans doute à remettre en cause les habitudes de lecture mobilisées (transférées ?) pour les autres textes.

\subsection{Retour sur l'Expérimentation 3}

Nous avons essayé de montrer ci-dessus que dans le récit fictionnel littéraire lu par les informateurs, la reconstitution de la séquence d'actions ne pouvait pas être dissociée de la reprise du discours rapporté dans la deuxième macro-unité du texte (MU2).

Cependant, on observe également que dans aucun résumé, aussi bien en situation de lecture LM ou LE, il n'est fait allusion au fait que le réceptionniste ne répond pas directement à Sebastián dans tout le début de sa 1e intervention en P7; de plus le fait dans sa 2e intervention, Sebastián ne s'adresse pas directement au réceptionniste mais 'à toutes les pensions du monde' n'a jamais été relevé. Enfin, les décalages entre le récit présenté par Sebastán dans son 'réquisitoire' et les éléments de ce même récit dans 
MU1 et dans la 1e intervention de Sebastián en P6 n'ont jamais été mentionnés sous une forme quelconque. Tous les propos du réceptionniste et de Sebastián 'sont pris pour ce qu'ils sont' alors que le travail de l'écriture vise à détourner cet échange de ce à quoi il pourrait référer dans les expériences de la réalité \{la recherche d'une chambre\} d'un jeune Espagnol ou Français (ou des représentations qu'ils s'en font). En fin de compte, ces lecteurs ne parviennent pas à effectuer ce que W. Iser (1985: 190) appelle une 'dépragmatisation des normes' (il s'agit ici des normes sociales et culturelles).

Dans les résumés effectués en LM ou LE, l'absence totale d'éléments renvoyant d'une façon ou d'une autre à la manière dont MU2 est mise en texte par l'écriture, renverrait

105 - soit à une certaine expérience de la textualité, d'ailleurs partagée par les élèves espagnols et français: un texte (même littéraire) étant d'abord considéré comme référant à une certaine réalité dont il serait purement et simplement le reflet. L'âge des informateurs pourrait alors fournir un élément d'explication : à cette étape de leur développement, ce serait la représentation du littéraire la plus prégnante ;

106 - soit à la situation de lecture dans laquelle se sont trouvés nos informateurs : texte lu en classe, imposé de l'extérieur, sans qu'il y ait véritablement projet de lecture ou un engagement personnel de leur part ;

107 - soit à la nature de l'épreuve de vérification de la compréhension utilisée. Le résumé (en tant qu'exercice scolaire) ne permettrait pas à l'élève d'exprimer la prise en compte de phénomènes directement en relation avec le travail de l'écriture dans le texte. On peut penser que si d'autres modes de questionnement avaient été mis en œuvre, des réponses allant dans ce sens auraient pu être obtenues. On retrouve ici posé le problème de la nature des épreuves utilisées pour accéder au résultat de l'activité de compréhension.

108 Il est vraisemblable que les résultats obtenus s'expliquent par la conjonction de ces différentes explications.

109 L'Expérimentation 3 pose directement le problème de la relation entre la compétence textuelle et le littéraire. Nous estimons qu'on ne saurait exclure le littéraire d'une réflexion sur la compétence textuelle. Notre position sur cette question est en fait très proche de celle de Jakobson (1963) concernant le poétique: de la même façon que la fonction poétique est une des fonctions fondamentales du langage, on pourrait dire que le littéraire est une des composantes essentielles de la compétence textuelle. Pour nous, l'expérience du littéraire fait partie (à des degrés divers) de l'expérience des textes acquise par les lecteurs alloglottes avec lesquels nous travaillons. De la même façon que la fonction poétique se manifeste dans les usages quotidiens du langage, nous pensons que les préoccupations de 'forme' (présentation du texte, 'style', quel que soit le sens qu'on donne à ce terme) sont présentes dans tous les écrits y compris les 'documents'. Il y a une dimension esthétique, de l'expérience de la textualité qui pour certains lecteurs joue un rôle important $t^{15}$. De ce point de vue, on pourrait dire que la littérature, explore et pousse parfois dans leurs limites des potentialités déjà inscrites dans la manière dont la textualité prend corps dans une langue-culture donnée.

L'expérience du littéraire est indissociablement liée à une expérience du langage écrit dans laquelle se conjuguent la prise en compte de sa dimension sociale (et par conséquent culturelle) et de sa dimension esthétique avec l'appréciation du travail de l'écriture dans le texte ('le style'). 
111 C'est pourquoi pour nous, les recherches en lecture-compréhension de textes se doivent de prendre en compte le fait littéraire. Le caractère fictionnel de la plupart des textes littéraires renvoie au problème de la fiction et de ce qu'elle induit dans la relation émetteur - texte - lecteur (rappelons à ce sujet que les énoncés fictionnels occupent aujourd'hui une place importante dans la pragmalinguistique ${ }^{16}$. Mais le littéraire pose aussi le problème de la cohérence d'une manière à nos yeux différente de la théorie de la pertinence. Si l'on revient à l'Expérimentation 3, la cohérence du texte se construit à partir de la déconstruction d'une représentation de la réalité évoquée, ce qui fait que les calculs inférentiels induits par cette représentation s'avèrent insuffisants.

5. Conclusion

112 Les différentes expérimentations dont nous avons rendu compte, effectuées à partir de lectures de textes en LM et en LE, permettent de préciser certains aspects de la compétence textuelle mise en œuvre.

113 Au niveau de la catégorisation des textes, il apparaît que chaque texte est exploré à la fois en tant que représentant d'un genre et à la fois en tant qu'exemplaire unique de ce genre. On retrouve là la double dimension du genre signalée par Bronckart.

$114 \mathrm{Au}$ niveau de la présence du discours rapporté, on notera qu'elle semble retenir fortement l'attention des lecteurs. Ceci serait confirmé par l'analyse de la localisation des soulignements. De plus, si l'on regarde ce qui se passe dans les épreuves de vérification de la compréhension du type rappel ou résumé, on s'aperçoit que la présence du discours rapporté est prise en compte et qu'elle intervient directement, qu'il s'agisse de la reconstruction de l'information dans un fait divers journalistique ou de la reconstitution de l'enchaînement des actions dans un récit fictionnel-littéraire.

115 On peut donc considérer que ce phénomène discursif particulier fait partie de la compétence textuelle acquise par les lecteurs. Il s'agit là d'une observation pour nous intéressante. Nous avons constaté en effet que l'existence de la polyphonie dans un texte avec la multiplication des repérages énonciatifs qu'elle induit est rarement mentionnée comme source de difficultés supplémentaires dans les verbalisations recueillies après la lecture en situation LM ou LE.

116 On s'aperçoit cependant que ce constat ne nous dit rien des transferts possibles de la L1 à la L2. Le texte thaï de l'Expérimentation 2 pose clairement la question de la nature culturelle/religieuse du discours rapporté qui semble avoir eu une incidence forte au niveau des rappels y compris en L1. Quant aux résultats de l'Expérimentation 3, ils renvoient à la non prise en compte du travail de l'écriture dans la mise en texte. C'est le rapport du lecteur au littéraire qui est posé. Toutefois, les résumés obtenus ne permettent pas d'avancer d'hypothèse fiable à ce sujet.

117 Laboratoire 'Geste, Parole, Scripturalité et Didactique des langues' - Centre de Linguistique Appliquée - 6, rue Gabriel-Plançon, F25030 Besançon Cedex, mél: marc.souchon@univ-fcomte.fr 


\section{BIBLIOGRAPHIE}

ALDERSON, J.C. \& A.H. URQUHART. 1984. Reading in a Foreign Language. Longman, New-york (4e édition 89).

BARTHES, R. 1973. Le Plaisir du texte. Seuil, coll. Points, Paris.

BRONCKART, J.-P. 1994. Lecture et écriture : éléments de synthèse et de prospective. In Reuter, Y. (Dir), Les interactions lecture-écriture, Actes du Colloque Théodile-Crel, Peter Lang.

BRONCKART, J.-P. 1996. Activité langagière, textes et discours. Delachaux et Niestlé, Lausanne, Paris.

CHAROLLES, M. 1978. Introduction aux problèmes de la cohérence des textes. In Langue française, $\mathrm{n}^{\circ} 38,7-41$.

DABENE, M. 1991. Un modèle didactique de la compétence scripturale. In Repères, $n^{\circ}$ 4, 9-22, I.N.R.P., Paris.

GAONAC'H, D. 1987. Théories d'apprentissage et acquisition d'une langue étrangère. Hatier, coll. LAL, Paris.

ISER, W. 1985. L'acte de lecture. Théorie de l'effet esthétique. Mardaga, Liège (traduit de l'allemand, ed. originale 1976).

JAKOBSON, R. 1963. Essais de linguistique générale. Paris. Éditions de Minuit.

KAMPANTHONG, O. 1993. Compétence de lecture en langue maternelle et en langue française chez les étudiants thaillandais, thèse de Doctorat, Université Stendhal - Grenoble III.

MOESCHLER, J. \& A. REBOUL. 1994. Dictionnaire encyclopédique de pragmatique. Seuil, Paris.

PEYTARD, J. 1968. Problème de l'écriture du non-verbal dans le roman contemporain. In La Nouvelle critique, $\mathrm{n}^{\circ}$ spécial : Linguistique et littérature, colloque de Cluny, 29-35.

PEYTARD, J. et al. 1982. Littérature et classe de langue. Hatier, coll. LAL, Paris.

PEYTARD, J. \& S. MOIRAND. 1992. Discours et enseignement du français. Hachette, coll. Repères, Paris.

REBOUL, A. \& J. MOESCHLER. 1998. La pragmatique aujourd'hui. Une nouvelle science de la communication. Seuil, coll. Points, Paris.

SEARLE, J.R. 1979. Sens et expression. Éditions de Minuit, Paris.

SOUCHON, M. 1992. Lire entre langues et discours. Analyse des obstacles à la lecture de textes en langue étrangère. Travail effectué en vue de l'H.D.R., Faculté des Lettres de Besançon.

SOUCHON, M. 1995a. Problèmes posés par l'analyse de la reconstruction du sens dans la lecturecompréhension en langue étrangère. In Revue de Phonétique Appliquée, $\mathrm{n}^{\circ} 115$ - 116 - 117, 357 368.

SOUCHON, M. 1995b. Pour une approche sémiotique de la lecture en langue étrangère. In Semen, n¹0 Sémiotique(s) de la lecture, Annales Littéraires de l'Université de Besançon, Diffusion les Belles Lettres, Paris, 103 - 161.

SOUCHON, M. 1998. L'appréhension de la relation scripteur-lecteur à partir de l'analyse des résumés d'un même texte fictionnel-littéraire produits en situation LM et LE. In M. Souchon (Dir), Actes du Xe Colloque international «Acquisition d'une langue étrangère : perspectives et 
recherches», 507 - 517. Université de Franche-Comté, Centre de Linguistique Appliquée de Besançon.

\section{ANNEXES}

\section{ANNEXE 1}

Expérimentation 1 : localisation des soulignements dans 2 récits fictionnels littéraires de ETA (cf. section 3.1.)

\section{- TF8 Maman et son dîner}

P1 « Il n’y a rien pour le dîner, ce soir... P2 Ce matin, Tricotet n'avait pas encore “tué... P3 Il devait tuer à midi. P4 Je vais moi-même à la boucherie, comme je suis. P5 Quel ennui ! P6 Ah ! pourquoi mange-t-on ? P7 Qu'allons-nous manger ce soir?» P8 Ma mère est debout, découragée, devant la fenêtre. P9 Elle nous regarde, tour à tour, sans espoir. P10 Elle sait qu'aucun de nous ne lui donnera un avis utile. P11 Consulté, papa répondra :

« Des tomates crues avec beaucoup de poivre.

- P12 Des choux rouges au vinaigre, dira Achille, l'aîné de mes frères.

- P13 Un grand bol de chocolat ! » demandera Léo, le second.

P14 Et je réclamerai, en sautant en l'air parce que j'oublie souvent que j'ai quinze ans passés :

« Des pommes de terre frites! Des pommes de terre frites! Et des noix avec du fromage!»

P15 Mais il paraît que frites, chocolat, tomates et choux rouges ne « font pas un dîner »...

P16 « Pourquoi, maman?

- P17 Ne pose donc pas de questions stupides »

P18 Elle a déjà EMPOIGNÉ le panier fermé, en ROTIN noir, et s'en va, comme elle est.

COLETTE La maison de Claudine

Symboles utilisés : Rang 1 (R1) PETITES MAJUSCULES, R2 PETITES MAJUSCULES, R3 Italiques, R4 Italiques, R5 Souligné, R6 caractères 9 , R7 caractères 9

Nombre de lecteurs: 171 - Valeurs : R1 = :88 soulignements ; R2, 77 ; R3, 50 ; R4, 49 ; R5, 42 ; R6, 39 ; R7, 38.

\section{- TF16 Projets d'avenir}

P1 Peu après le patron m'a fait appeler et, sur le moment, j'ai été ennuyé parce que j'ai pensé qu'il allait me dire de moins téléphoner et de mieux travailler. P2 Ce n'était pas cela du tout. P3 Il m'a déclaré qu'il allait me parler d'un projet encore très vague. P4 Il voulait seulement avoir mon avis sur la question. P5 Il avait l'intention d'installer un bureau à Paris qui traiterait ses affaires sur la place, et directement, avec les grandes compagnies et il voulait savoir si j'étais disposé à y aller. P6 Cela me permettrait de vivre à Paris et aussi de voyager une partie de l'année. P7 «Vous êtes jeune, et il me semble que c'est une vie qui doit vous plaire » P8 J'ai dit que oui mais que dans le fond cela m'était égal. P9 Il m’a demandé alors si je n'étais pas intéressé par un changement de vie. P10 J'ai répondu qu'on ne changeait jamais de vie, qu'en tout cas toutes SE VALAIENT que LA MIENNE ici ne me déplaisait pas du tout. P11 Il a eu l'air mécontent, m'a dit que je 
répondais toujours à côté, que je n'avais pas d'ambition et que cela était désastreux dans les affaires. P12 Je suis retourné travailler alors. P13 J'aurais préféré ne pas le mécontenter, mais je ne voyais pas de raison pour changer ma vie. P14 En y réfléchissant bien, je n'étais pas malheureux.

\section{A. CAMUS L'Étranger}

Nombre de lecteurs : 118 - Valeurs:29, 23, 22, 21, 20, 19, 17

ANNEXE 2

Texte concernant l'Expérimentation 2 (cf. section 3.2.1.)

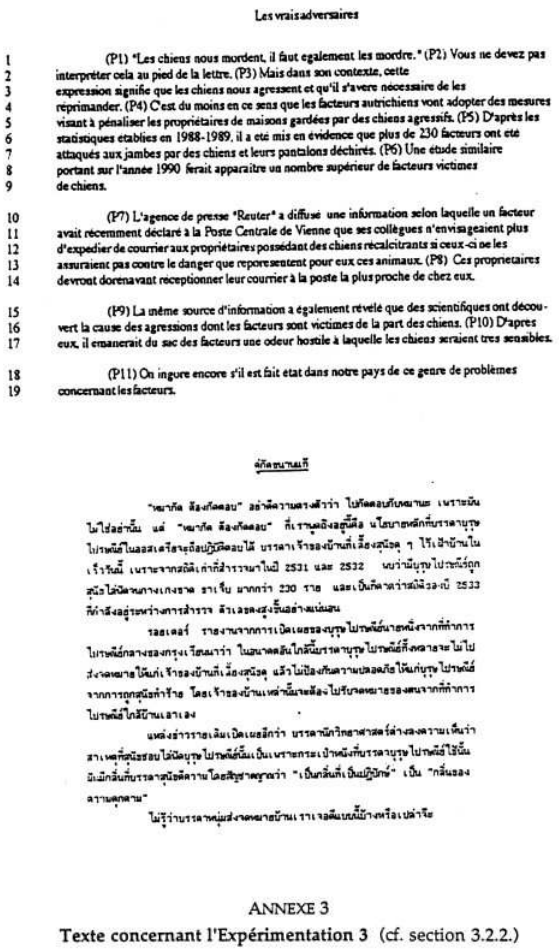

Un estudiante francés en Barcelona

Leg6 a Barcelons en la noche del veintisiete de julio y llovia. Bajó del tren y al ver en su reloi que eran las once de la noche. se convenctó de que lendría que dormir en la calle. Al salir de la estactón empezaron a aparecer ante sus oios los letreros quie enunciaben lás pensiones. los
hosinlns. Ins nlberguns. Sn diin : "No hay habitación pare usted" en la puerts de cunt:o pensinnes. pern se arrojó valientemente sobre la escalera que conducia a la quinta pensión que encontró. Perdió y volvió a encontrar su pasaporte enles de entrar. y luego avanzó hasta una especie de mostrador donde un recepcionista le podría estar confundiendo con un contrabandista .Quería de rodilles un cuarto para varios días porque en Bercelona se ibe a encontrar con los Linares. porque estaba muy resíriado y porcue lopielerio de ese pensión, el dueño de todos los cuartos de esa pensión de todas las mesas del comedor de esa pensión y después le dịio que no habia nada para ét. que sólo habia un cuerto con dos camas pera dos personas. Sebastián inició la más grnnde requisitoria contra todas las pensiones del mundo : a è que era ur estadiante extranjero. a tl que estaba regia (lo perdió y lo volvió a encontrar). a él que venía en busca de
descanso, de sol y del Quijote, sa lo recihfía con lluvia y se la obligaba* a dormir en la intemperie. "Calma. calma, señor. dijo el propietariopensión y le voy a conseguir un cuarto".

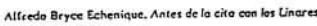

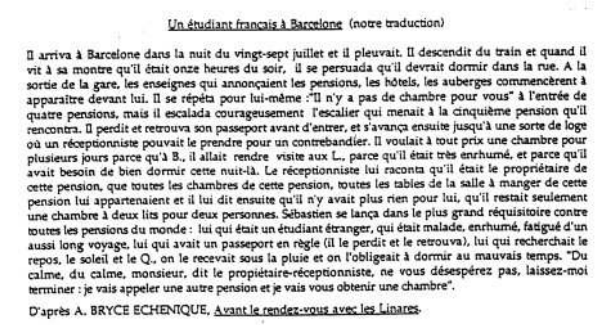




\section{NOTES}

1. Nous utiliserons ici « genre » dans le sens où J.P. Bronckart 1996 définit ce terme dans l'ouvrage déjà cité. L'opposition fondamentale qu'il établit entre genres (ou catégories de textes) et types de discours est largement développée dans la deuxième partie de l'ouvrage, p. 137 - 151.

2. Voir à propos de cette expérimentation : Souchon, M. (1992 et 1995b).

3. Il s'agit des 4 faits divers/faits de société suivants : « La dictée de Pivot avait de l'estomac » (L'Est Républicain du $3-12$ - 89) ; « Après avoir tué sa femme, ses 2 enfants, une amie » (France Soir du $1-09-90)$; « Le studio dépotoir de la madone des bouts de ficelle » (Libération du $7-08-90)$; « Nouveau meurtre d'enfant dans l'Isère » (Le Figaro du $6-08-90$ ).

4. Pour les 4 récits, voir E. Wagner (1965) p. 38, p. 55, p. 77 et p. 112 ; pour les 3 textes descriptifs : p. 15, p. 63 et p. 105 ; pour le poème de Prévert, p. 49 ; pour le texte argumentatif, p. 67.

5. Les analyses factorielles de correspondance ont été effectuées au laboratoire MIS de la Faculté des Lettres de Besançon à partir du logiciel ANACONDA.

6. Wagner, E. op. cit. p. 38.

7. Il est vraisemblable que parmi les raisons qui ont motivé la sélection de cet extrait par les auteurs du recueil, l'utilisation de ce lexique a dû jouer un rôle déterminant.

8. Wagner, E. : op. cit. p. 112.

9. Nous nous référons ici au Chapitre 7 : Méthodologie et analyse des résultats du test de construction du sens en langue maternelle chez les étudiants thaïs et français et en langue française chez les étudiants thaïs (p. 284-335).

10. Nous avons également consulté plusieurs informateurs thaïs.

11. Pour une analyse plus détaillée de ces résumés, on se référera à SOUCHON, M. (1995a).

12. Pour une analyse plus détaillée voir SOUCHON, M. (1998).

13. Souchon, M. (1992: 919-921).

14. Ce pourcentage a été calculé de la façon suivante : nombre total d'unités soulignées dans le texte divisé par le nombre de lecteurs x par le nombre d'unités du texte ramené à 100. Pour le texte qui vient en tête (que nous avons nous-même sélectionné), ce pourcentage est de 7, 21 ; pour le poème de Prévert, il est de 5,24.

15. Nous nous référons bien entendu au texte célèbre de Barthes (1973).

16. Nous signalerons entre autres Searle, J.R. (1979) ; Moeschler, J. \& A. Reboul (1994) notamment les chapitres 15 et 16 ; Reboul, A. \& J. Moeschler (1998).

\section{RÉSUMÉS}

La compétence textuelle a d'abord été plutôt envisagée du point de vue de la production (voir Charolles $1978: 8$ ). Nous l'aborderons ici uniquement du point de vue de la compréhension, à la lumière de recherches empiriques portant principalement sur la lecture de textes écrits en LE. Notre hypothèse comprend trois volets : (1) la compétence textuelle se constituerait à travers les 
diverses expériences de la textualité acquises par le sujet. (2) C'est en s'appuyant essentiellement sur sa compétence textuelle acquise que le sujet développerait des compétences de lecture en LE. (3) La lecture de textes en LE apparaît comme un lieu d'observation permettant de mieux appréhender les diverses composantes de la compétence textuelle

The notion of textual skills was first considered from the perspective of production (Charolles 1978: 8). In this paper, textual skills are examined exclusively from the standpoint of comprehension and in the light of empirical research in reading written texts in a foreign language. The author proposes a three-fold hypothesis : (1) textual skills are acquired through the reader's various experiences with text ; (2) the reader uses acquired textual competence as a basis for developing reading skills in a foreign language; and (3) the various elements which compose reading skills can be better understood by observing how texts are read in a foreign language

INDEX

Mots-clés : compétence textuelle, texte, genre, discours rapporté, le littéraire

\section{AUTEUR}

MARC SOUCHON

Université de Franche-Comté 